\title{
PERIPARTUM HYSTERECTOMIES AT THE KORLE-BU TEACH - ING HOSPITAL: A REVIEW OF 182 CONSECUTIVE CASES
}

\author{
"R.A. KWAME-ARYEE, A.K. KWAKYE, and J.D. SEFFAH \\ Department of Obstetrics and Gynaecology, Korle Bu Teaching Hospital, P O Box 82, \\ Korle Bu, Accra, Ghana
}

\begin{abstract}
SUMMARY
Objectives: The main objectives of this study were to determine the incidence, maternal characteristics, indications, the maternal and perinatal outcomes and the complications of emergency peripartum hysterectomies done at the Korle-Bu Teaching Hospital.

Study design: This is a retrospective descriptive study covering the period January 1, 2000 to June 30, 2003.

Setting: Maternity Unit of the Korle-Bu Teaching Hospital

Results: The incidence of peripartum hysterectomy during the study period was $4.34 / 1000$ deliveries. The main indication was severe uterine bleeding from uterine rupture 89 (48.9\%), uterine atony $60(33 \%)$, placenta accreta $20(11 \%)$ and post caesarean uterine wound sepsis and necrosis $11(6 \%)$.

The average blood loss was 2.02 (SD 0.86) $\mathrm{L}$ and all patients received blood transfusion. The mean age of the patients was 31.6 (SD 5.3) years and the mean parity was 2.7 (SD 1.7). Complications occurred in $28(19.8 \%)$ patients.

The stillbirth rate was 483.5/1000 deliveries and the maternal mortality was $12.9 \%$. The average duration of hospital stay was 11 (SD 8) days.

Conclusions: The incidence of emergency peripartum hysterectomy is high. It is performed for young nulliparous women through to elderly grand-multiparous women. The surgery is associated with significant maternal and perinatal morbidity and mortality. Timely expert management in a well resourced centre is advocated.
\end{abstract}

Keywords: Peripartum, hysterectomy, uterine rupture, uterine atony, maternal morbidity, maternal mortality.

\section{INTRODUCTION}

Emergency peripartum hysterectomy is one of the life saving surgical procedures performed at the Maternity Unit of the Department of Obstetrics and Gynaecology of the Korle-Bu Teaching Hos- pital. Life-threatening haemorrhage resulting from uterine rupture and atony has become rare events in the developed world ${ }^{1}$ but they continue to pose a major problem in obstetric care in developing countries.

The modern era of caesarean hysterectomies begun in the 1940's and with improvement in anaesthetic techniques the indication widened to include elective sterilization. Several reports have confirmed the usefulness of peripartum hysterectomy for emergency cases of haemorrhage as well as for other gynaecological conditions complicating pregnancy $^{2,3}$. No work has yet been done to determine the role of peripartum hysterectomy in obstetric care and its associated morbidity and mortality at the Korle-Bu Teaching Hospital.

The aim of this study was to determine the incidence of peripartum hysterectomy, the indications, the perinatal and maternal outcomes and the complications associated with the procedure at the Korle-Bu Teaching Hospital.

\section{PATIENTS AND METHODS}

This was a retrospective descriptive study in which the hospital records of all the patients who underwent emergency peripartum hysterectomy from January 1, 2000 to June 30, 2003 were reviewed. Peripartum hysterectomy was defined as hysterectomy performed at the time of delivery or within forty-two days of delivery. The hysterectomies were performed by Consultants (assisted by their Residents or Senior Residents) and Senior Residents (assisted by their Consultants or other Residents). There were minor differences in operating technique among the surgeons.

The age, parity, indication for the hysterectomy, estimates of blood loss, type of hysterectomy, maternal and perinatal outcomes, duration of hospital stay and complications were obtained from the records.

${ }^{*}$ Author for correspondence
kwamearyee@yahoo.com 
All the patients received prophylactic antibiotics peri-operatively. Most of the patients were managed postoperatively in the recovery ward. The few seriously ill patients who required intensive care were transferred to the Intensive Care Unit.

Records of blood loss available were not limited to blood loss during hysterectomy but included blood loss before the procedure.

Febrile morbidity was defined as a temperature of $38^{\circ} \mathrm{C}$ or more on any two consecutive days after surgery but excluding the first day. Wound infection was defined as the presence of any two of the following: purulent discharge and/or obvious cellulitis, elevated temperature, and positive wound culture.

The results obtained were analyzed with the SPSS 10.0 for Windows and presented in the form of descriptive statistics.

\section{RESULTS}

There were 182 peripartum hysterectomies during the three-and-a half-year period from January 1, 2000 to June 30, 2003. During this same period there were a total of 41,978 deliveries. The peripartum hysterectomy rate, therefore, was 4.34/1,000 deliveries.

Table 1 Age distribution of patients

\begin{tabular}{|c|c|}
\hline Age & Number (\%) \\
\hline $16-20$ & $3(1.7)$ \\
\hline $21-25$ & $27(14.8)$ \\
\hline $26-30$ & $49(26.9)$ \\
\hline $31-35$ & $56(30.8)$ \\
\hline $36-40$ & $41(22.5)$ \\
\hline $41-45$ & $5(2.7)$ \\
\hline$\geq 46$ & $1(0.6)$ \\
\hline Total & $182(100)$ \\
\hline
\end{tabular}

The ages of the patients ranged from 16-46 years with a mean of 31.6 (SD 5.3) years (Table1). The highest frequency was in the 31-35 year group followed by the $26-30$ year group. Together the 2 groups constitute about $58 \%$ of the total population.

The parity of the patients ranged from $0-8$ with a mean of 2.7 (SD 1.7) (Fig. 1). The highest frequency was in those who were para 2. A little over $70 \%$ of the patients were para 3 or less.
The indication for peripartum hysterectomy was severe haemorrhage caused by ruptured uterus 89 $(48.9 \%)$; uterine atony $60(33.0 \%)$; placenta accreta $20(11.0 \%)$ and post caesarean section uterine wound sepsis and necrosis $11(6.0 \%)$ (Table 2). Two patients $(1.1 \%)$ had hysterectomy for postpartum haemorrhage in a fibroid uterus.

Table 2 Causes of bleeding necessitating peripartum hysterectomy

\begin{tabular}{llcc} 
Cause of bleeding & $\begin{array}{c}\text { Num- } \\
\text { ber }\end{array}$ & Percentage \\
\hline 1. & Ruptured Uterus & 89 & 48.9 \\
2. Uterine Atony & 60 & 33.0 \\
3. Placenta Accreta & 20 & 11 \\
4. Post Caesarean & 11 & 6.0 \\
$\quad$ Section Uterine & & \\
$\quad$ Wound Sepsis and & & \\
$\quad$ Necrosis & 2 & 1.1 \\
5. Uterine Fibroids & $\mathbf{1 8 2}$ & $\mathbf{1 0 0}$ \\
\hline Total & &
\end{tabular}

There were only $4(4.5 \%)$ cases of rupture in a scarred uterus that required hysterectomy. The patients with atonic uterus had some conservative methods of treatment before the final decision to perform a hysterectomy. These included the use of oxytocin drip (40 units per litre of saline), administration of intravenous ergometrine $0.25-0.5 \mathrm{mg}$ and rectal misoprostol $800 \mu \mathrm{g}$.

Figure 1 Parity of patients

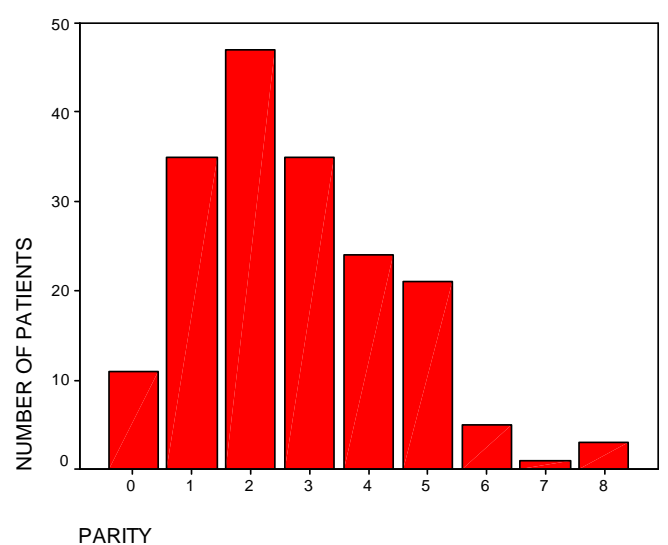

After hysterectomy were the following conditions: normal delivery 47 (25.8\%); caesarean delivery 84 (46.2\%); laparotomy for ruptured uterus 48 (26.4\%); vacuum extraction $2(1.1 \%)$ and breech extraction $1(0.5 \%)$. 
One hundred and seventy $(93.4 \%)$ of the hysterectomies were total and $12(6.6 \%)$ were subtotal. The reasons for the sub-total hysterectomy among these few patients were not stated.

Estimated blood loss ranged from 0.8 to $4.5 \mathrm{~L}$ with a mean blood loss of 2.02 (SD 0.86) L. All the patients received blood transfusion of 2-5 units. All the patients received perioperative antibiotics comprising ampicillin, gentamicin and metronidazole.

Complications occurred in $28(19.8 \%)$ of the patients (Table 3 ). None of the patients with complications was screened for HIV infection.

Table 3 Complications of Peripartum Hysterectomy

\begin{tabular}{llc} 
& \multicolumn{1}{c}{ Complication } & Number $(\%)$ \\
\hline 1. & Bladder Injury & $8(4.4)$ \\
2. & Wound Sepsis & $6(3.3)$ \\
3. & Intensive Care & $5(2.7)$ \\
4. & Febrile morbidity & $4(2.2)$ \\
5. & Broad ligament haema- & $2(1.1)$ \\
& toma & $1(0.5)$ \\
6. & Cuff cellulites & $1(0.5)$ \\
7. & Pelvic abscess & $1(0.5)$ \\
8. & Vesico-vaginal fistula & $\mathbf{2 8 ( 1 9 . 8 )}$ \\
\hline \multicolumn{2}{l}{ Total }
\end{tabular}

Twenty-two of the patients died of various causes within 48 hours of surgery. The case fatality rate was therefore $12.9 \%$. The mortality was distributed as follows: uterine atony - $13(59.1 \%)$; ruptured uterus - $6(27.3 \%)$; placenta accreta - 2 $(9.1 \%)$ and secondary postpartum haemorrhage - 1 $(4.5 \%)$.

There were 94 live births and 88 stillbirths. The stillbirth rate was 483.5 per1000 deliveries. Sixtysix $(74.2 \%)$ of the stillbirths occurred in cases of uterine rupture whilst $18(25.8 \%)$ occurred in cases of uterine atony. Forty-two (70\%) of the live births occurred in cases of uterine atony and $23(25.8 \%)$ occurred in cases of uterine rupture. The live births seen in cases of uterine rupture were mostly among patients who had had normal delivery but were later found to have a ruptured uterus.

The mean duration of hospital stay in survivors was 11 (SD 8) days with a range of 5-42 days. The longest duration of hospital stay of 42 days occurred in a patient who developed severe sepsis and a pelvic abscess following surgery.

\section{DISCUSSION}

This is a retrospective study of women who underwent peripartum hysterectomy at the Korle $\mathrm{Bu}$ Teaching Hospital between $1^{\text {st }}$ January 2000 and $30^{\text {th }}$ June 2003 . The study design was limited by insufficient data in a few cases.

Peripartum haemorrhage is a common obstetric problem encountered at the Korle-Bu Teaching Hospital and it is also a major cause of maternal morbidity and mortality. Haemorrhage, the leading cause of maternal mortality, during the ten-year period of 1984-1994, represented $17.75 \%$ of all maternal deaths ${ }^{4}$ Emergency hysterectomy is a recognized means of controlling peripartum haemorrhage. During the study period the hysterectomy rate for life-threatening obstetric haemorrhage was 4.34/1000 deliveries which is high compared to 1.2/1000 deliveries in King Edward VIII Hospital in Durban, South Africa ${ }^{5}$ and 1.22/1000 from an inner city hospital in Saudi Arabia ${ }^{6}$. These differences in the incidence of peripartum hysterectomy may be explained by the different levels of sophistication in obstetric health care and the patient load.

The mean age of 31.6 years is similar to the findings of Sebitloane in South Africa ${ }^{5}$. The youngest patient in this study was 16 years old; she had hysterectomy for severe atonic uterine haemorrhage that was unresponsive to conservative measures.

The major indications for peripartum hysterectomy in this study were ruptured uterus, uterine atony, placenta accreta and secondary postpartum haemorrhage. This is consistent with the findings from Ga-Rankuwa Hospital in South Africa ${ }^{7}$. Atonic postpartum haemorrhage followed by ruptured uterus was noted by Nasrat ${ }^{6}$. The indications for peripartum hysterectomy in some countries have changed over the years to reflect the improvement in obstetric care; in these countries uterine rupture has been eliminated completely from the list of indications ${ }^{8}$.

Secondary postpartum haemorrhage in patients who had been delivered previously by caesarean section constituted $6 \%$ of the indications for hysterectomy in this study. The haemorrhage in these cases resulted from sepsis and necrosis of the uterine wound. Sepsis was also a significant indication for peripartum hysterectomy in the studies by Sebitloane ${ }^{5}$ and Shava ${ }^{7}$ and it has been suggested that these cases of sepsis were the result of compromised immunity. In the study by Sebitloane 
nine out of ten patients with sepsis who gave their consent for HIV testing were positive ${ }^{5}$. None of our patients who developed sepsis after emergency hysterectomy was screened for HIV infection.

One hundred and seventy (93.4\%) patients had a total abdominal hysterectomy with the remaining $12(6.6 \%)$ having a sub-total hysterectomy. It has been suggested that the routine performance of emergency total abdominal hysterectomy is unnecessary and potentially harmful ${ }^{9}$. Subtotal hysterectomy is used routinely in some centres for uterine rupture requiring hysterectomy ${ }^{10}$. At the Korle-Bu Teaching Hospital emergency hysterectomies are usually total as shown in this study. Murta et $\mathrm{al}^{11}$ found that there were no statistically significant differences between subtotal and total hysterectomy regarding operating time, need for transfusion, number of intra- and post-operative complications and maternal mortality. Advocates of total hysterectomy have cited the long-term complications of vaginal discharge, acyclic bleeding and the need for cervical cytology as reasons for the choice of total hysterectomy. Other reasons to support total hysterectomy are the high cervicovaginal infection rates and the high incidence of advanced carcinoma of the cervix in women in developing countries who do not have access to periodic pap smears.

The maternal mortality of $12.9 \%$ is high compared to the $0 \%$ and $4.5 \%$ reported in other studies ${ }^{3,8}$. Uterine atony and ruptured uterus accounted for 13 $(59 \%)$ and $6(27.2 \%)$ of the deaths, respectively. The response to ruptured uterus in Korle- $\mathrm{Bu}$ has always been swift and there has been no hesitation about the decision to operate. Problems arise when dealing with atony of the uterus; the diagnosis is made when conservative measures have failed to control bleeding by which time the patient may have lost a large volume of blood and her condition may be in extremis. In our unit uterine atony is managed initially with manual massage of the uterus, administration of intravenous oxytocin and ergometrine supplemented by the replacement of blood volume with crystalloids, colloids and blood transfusion. Prostaglandin $\mathrm{F}_{2} \alpha$ and rectal misoprostol have also been used.

Surgery is considered when these conservative measures have failed. Other considerations like low parity may contribute to the delay in performing a hysterectomy. These delays coupled with the limited supplies of blood and blood products may have contributed to some of the adverse outcomes associated with uterine atony in this study. Early decision making and intervention may help reduce the high mortality associated with uterine atony. The use of hysterectomy to control bleeding in young women of low parity is undesirable and other methods like the B-Lynch brace procedure ${ }^{12}$, ligation of the uterine arteries and ligation of the internal iliac arteries may be used in selected cases to control atonic uterine haemorrhage. Ligation of the internal iliac arteries requires the services of a skilled surgeon and may not be the procedure of choice in the setting of massive haemorrhage and an unstable patient as it may be associated with a substantial risk of failure ${ }^{13}$. The B-Lynch procedure and ligation of the internal iliac arteries are occasionally used in our unit but no studies have been done to assess the effectiveness of these methods in our practice.

Packing of the uterus in severe atonic uterine haemorrhage has received both favourable ${ }^{14}$ and unfavourable ${ }^{15}$ reviews as one of the methods of controlling uterine haemorrhage. Concerns have been raised about its tendency to conceal continued bleeding, uterine trauma and infection. Some authors are of the view that these concerns have been overestimated ${ }^{16}$. Uterine packing is not routinely used in our unit.

Hiyashi et al have reported the successful control of severe postpartum haemorrhage with a prostaglandin $F_{2} \alpha$ analogue ${ }^{17}$. This product is also not available for routine use in our labour wards.

Complications from peripartum hysterectomy are high because of the increased blood supply to the pelvic organs during pregnancy, the distorted pelvic anatomy as a result of the enlarged uterus and the fragility of the tissues ${ }^{18}$. Twenty-eight $(19.8 \%)$ of our patients had complications and this compares well with other studies ${ }^{5,8}$. The incidence of morbidity is influenced by many factors including the indication for surgery and the use of perioperative antibiotics. Antibiotics are given to all patients who undergo peripartum hysterectomy at the Maternity Unit. There was a high prevalence of infectious morbidity in the complications noted; we may have to study in detail our choice of postoperative antibiotics and the incidence of septic complications with the view to altering our antibiotics from the routine combination of ampicillin, gentamicin and metronidazole to more powerful ones. Currently co-amoxyclav and cephalosporins such as cefuroxime and ceftriaxone are gradually replacing the ampicillin and gentamicin. 
The stillbirth rate of $483.5 / 1000$ is high but not surprising as $72.4 \%$ of these deaths were a direct result of ruptured uterus. The stillbirth rate from ruptured uterus was $74.2 \%$ and this is comparable to the $73.4-80 \%$ obtained by Adanu ${ }^{21}$ and Sebitloane . $^{5}$.

\section{CONCLUSION AND RECOMMEN- DATION}

Severe life-threatening haemorrhage requiring hysterectomy is not an uncommon event in obstetric practice in a developing country such as ours. The incidence of emergency peripartum hysterectomy is high in our institution. It is performed for young nulliparous women through to elderly grand-multiparous women. The main indication is life-threatening haemorrhage. The surgery is associated with significant maternal and perinatal morbidity and mortality.

It may be possible to anticipate haemorrhage in some situations but severe uncontrollable haemorrhage can occur unexpectedly. It is in these situations that early decision making and the provision of adequate supplies of blood and blood products become paramount.

All obstetricians should be adequately trained in the performance of the B-Lynch procedure, emergency hysterectomy and other complicated procedures such as ligation of the internal iliac arteries to control uterine haemorrhage. Efforts should be made to make available prostaglandins for the management of atonic postpartum haemorrhage. Finally clear protocols and drills on the management of peripartum haemorrhage may help reduce the incidence of peripartum hysterectomy.

\section{ACKNOWLEDGEMENT}

We wish to acknowledge the help given us by the Staff of the Records Department of the Department of Obstetrics and Gynaecology for painstakingly retrieving the old folders for us.

\section{REFERENCES}

1. Plauche WC. Caesarean hysterectomy: indications, technique, and complications. Clin $\mathrm{Ob}$ stet Gynecol. 1986; 29: 318.

2. Mokgokong ER, Marivate M. Treatment of ruptured uterus. Afr Med J 1976; 50: 162.

3. Gonsoulin W, Kennedy RT, Guidry KH. Elective versus emergency caesarean hysterectomy cases in a residency program setting: A review of 129 cases from 1984 to 1988. Am J Obstet Gynecol 1991; 65(1): 91-94.

4. Lassey AT, Wilson JB. Trends in maternal mortality in Korle-Bu Teaching Hospital. Ghana Med J 1998; 32: 910-916.

5. Sebitloane MH, Moodley J. Emergency peripartum hysterectomy. East Afr Med J 2001; 78(2): 70-74.

6. Nasrat HA. Near-miss obstetric morbidity in an inner city hospital in Saudi Arabia. East Mediterr Hlth J 1999; 5(4): 712-726.

7. Shava J, Masihleho GE, Mazibuko MD. Peripartum hysterectomy at Ga-Rankuwa Hospital: a two and a half year review. Cent Afr J Med 1996; 42(1): 25-28.

8. Zorlu CG, Turan C, Isik AZ, Danisman N, Mungan T, Gökmen O. Emergency hysterectomy in modern practice. Changing clinical perspective in time. Acta Obstet Gynecol Scand 1998; 77(2): 186-190.

9. Heij HA, Te Velde ER, Cairns JM. Management of rupture of the gravid uterus. Trop Doct 1985; 15: 127-131.

10. Megafu U. Factors influencing maternal survival in ruptured uterus. Int J Gynecol Obstst 1985; 23 : 475-480.

11. Murta FF, Carniero JG, De Frectas MM. Total hysterectomy versus subtotal hysterectomy: which procedure should be done during the pregnant-puerperal period? Rev Perinat Med 1993; 111: 354.

12. B-Lynch C, Coker A, Lawal AH, Abu J, Cowen MJ. The B-Lynch surgical technique for the control of massive postpartum haemorrhage: an alternative to hysterectomy? Five cases reported. Br J Obstet Gynaecol 1997; 104: 372-375.

13. Evans S, McShane P. The efficacy of internal iliac artery ligation in obstetric haemorrhage. Surg Gynecol Obstet 1985; 66: 89-92.

14. Drucker M, Wallach RC. Uterine packing: a reappraisal. Mount Sinai J Med 1979; 46: 191194. 
15. Day LA, Mussey RD, De Voe RW. The intrauterine pack for postpartum haemorrhage. Am J Obstet Gynecol 1948; 55: 231-243.

16. Katesmark M, Brown R, Raju KS. Successful use of a Sengstaken-Blakemore tube to control massive postpartum haemorrhage. Br J Obstet Gynaecol 1994; 101: 259-260.

17. Hayashi RH, Castillo MS, Noah ML. Management of severe postpartum haemorrhage with a Prostaglandin $\mathrm{F}_{2} \alpha$ Analogue. Obstet Gynecol 1984; 63: 806-808.

18. Barclay DL. Caesarean hysterectomy at the Charity Hospital in New Orleans- 1000 consecutive operations. Clin Obstet Gynecol 1969; 12: 635-637.
19. Clark SL, Yeh SY, Phelan JP, Bruce S, Paul RH. Emergency hysterectomy for obstetric haemorrhage. Obstet Gynecol 1984; 64(3): 376-380.

20. Chanrachakul B, Chaturachinda K, Phuapradit W, Roungsipragarn R. Caesarean and postpartum hysterectomy. Int J Gynecol Obstet 1996; 54: 109-113.

21. Adanu RMK, Obed SA. Ruptured uterus: A seven-year review of cases from Accra, Ghana. J Obstet Gynaecol Can 2003; 25(3): 225-230. 